\title{
INVESTIGATION OF DYNAMIC MOTION PROCESSES OF MODERNIZED UAV USING MATHEMATICAL MODEL OF NUMERICAL SIMULATION
}

\author{
VASSILIY KRYUCHKOV ${ }^{1}$, VITALY SAVINKIN ${ }^{2}$, ANDREY SOLODOVNIK ${ }^{3}$ \\ \& LYUDMILA KRYUCHKOVA ${ }^{4}$ \\ ${ }^{1}$ Research Scholar, Department of Transport and Mechanical Engineering, North Kazakhstan State University, \\ Petropavlovsk, Republic of Kazakhstan \\ ${ }^{2}$ Assistant professor, Head of the Department of Transport and Mechanical Engineering, \\ North Kazakhstan State University, Petropavlovsk, Republic of Kazakhstan \\ ${ }^{3}$ Professor, Department of Physics, North Kazakhstan State University, Petropavlovsk, Republic of Kazakhstan \\ ${ }^{4}$ Research Scholar, Department of Information and Communication Technology, North Kazakhstan State University,
} Petropavlovsk, Republic of Kazakhstan

\begin{abstract}
The article describes the modernization of Unmanned Aerial Vehicle (UAV) to perform observations of satellites. The technical requirements applied to the device and the parameters of the payload and its parameters are verified. Various models used for aerodynamic calculations are displayed and their pros and cons are revealed. Various methods of constructing solid-state models are also displayed. Several different shapes of the dome for equipment placement are proposed. The optimal shape of the dome was chosen based on the results of calculations on the pressure distribution on the body. The angle of attack was chosen based on the calculation of the resistance and lift forces.

KEYWORDS: UAV, Aerodynamics, Fuselage Modernization, SolidWorks, Forces \& Simulation
\end{abstract}

Received: Nov 02, 2019; Accepted: Nov 22, 2019; Published: Mar 17, 2020; Paper Id.: IJMPERDAPR202056

\section{INTRODUCTION}

According to the military doctrine of the Republic of Kazakhstan, modern military conflicts have been characterized by their activity, the brevity of the scale, spheres of warfare (space and information space) as well as high voltage strength and resources of the state in the armed struggle (Ministry of Defense of the Republic of Kazakhstan official resource). In this regard, it is of great importance for the defense of the country to get the direction associated with continuous spacecraft tracking and compiling information about them. Satellite radar is hampered by its small size and long distances. With this, we put forward the idea of monitoring these devices using photomultipliers, which allow us to study even faint objects. However, observations in the visible range from the earth's surface are often hampered by dense tropospheric clouds. Based on our analysis (Savinkin et al., 2018), it was revealed that statistically dense clouds are formed at altitudes not exceeding $3.5 \mathrm{~km}$.

Such heights are available to a wide range of aircraft and, in particular, unmanned aerial vehicles. Based on this, we see a solution to the problem of improving the efficiency of monitoring the upper sphere in the design (and possibly adaptation) of an unmanned aerial vehicle capable of rising to heights of up to $4000 \mathrm{~m}(4.0 \mathrm{~km}) \mathrm{with}$ the possibility of installing a container with scientific equipment. This container can be a dome camera with a gyro platform for panoramic shooting during flight. 
The study of quite a large number of different aircrafts led to the choice of the basic model of the UAV GeoScan 201, the characteristics of which are given below (Savinkin et al., 2018):

Max. takeoff weight: $8.5 \mathrm{~kg}$

Flight speed: $64-130 \mathrm{~km} / \mathrm{h}$

Flight duration: up to 3 hours

Max. pay load weight: $1.5 \mathrm{~kg}$

Max. flight altitude: $4000 \mathrm{~m}(4.0 \mathrm{~km})$

Operating temperature: $-40{ }^{\circ} \mathrm{C}$ to $+40{ }^{\circ} \mathrm{C}$

As can be seen from these characteristics, this device has a sufficient practical ceiling to overcome the dense tropospheric clouds and is also able to take quite a decent mass of payload, which allows us to modify this UAV for our tasks.

As a payload, we selected two video tracking devices - one camera with a viewing angle of $180^{\circ}$ for viewing the front hemisphere of the aircraft, installed in the front part of the body, and the second aimed at the zenith and equipped with a photomultiplier for tracking satellites installed in the middle part of the fuselage.

To justify the choice of the optimal aerodynamic shape of the dome, it is necessary to calculate the different types of dome on the base glider. For this purpose, various mathematical models describing the flow of subsonic air masses were chosen.

The Navier-Stokes equations are the basis for solving aerodynamics problems (Belotserkovsky et al., 2000; Belotserkovsky et al., 2001; Shevelev and Maksimov, 2000; Shevelev and Maksimov, 2003a; Shevelev and Maksimov, 2003b). However, solving the complete Navier-Stokes equations remains a very difficult task even for geometrically simple cases. Solving problems of external aerodynamics for real aircraft can be done only with the use of specific simplifications. Previous researchers - Belotserkovsky O. M., Andrushchenko V. A. and Shevelev Yu. D. used three different methods of solving the Navier-Stokes equations as a mathematical model for the study of viscous incompressible gas flow near bodies of complex shapes: (1) the method of "artificial" compressibility, (2) the method of splitting by physical variables, generalized to the case of arbitrary curvilinear coordinates and (3) the method of calculating the Navier-Stokes equations in the "thin" layer approximation. However, in the proposed mathematical model, Belotserkovsky, O. M., Andrushchenko, V. A. and Shevelev, Yu. D.'s emphasis is placed on the principles of distribution of velocity $V x, V y, V z$, and the pressure of the flow medium impact $p x, p y, p z$ does not take into account the dynamic impact of body parts and feature of the layout of equipment on the aircraft. The expansion of the functionality of the developed aircraft, such as the ability to observe the upper hemisphere of the outer space as well as the tracking of natural objects, inevitably entails a structural change that affects the dynamic loads, namely the air resistance forces $F_{r}$ acting on the apparatus. Therefore, there is a need to improve the mathematical model of the study of the distribution of forces and pressure, taking into account the dynamic and structural changes of the apparatus. Also, the task of mathematical modeling is complicated by the fact that a significant role in the adequacy of the model is played not only by established processes, but also by transients in the boundary layers of interaction of the contact medium with the object of study, for example, in the approximation of a thin layer. 
To study the pitch of an unmanned aerial vehicle, it is important to describe mathematically the process of flows of viscous compressible gas near bodies of non-regular shape. For this purpose, various methods of calculation of stationary and non-stationary, three-dimensional Euler and Navier-Stokes equations written in conservative form are used. To solve the scientific and technical problem, several solutions are proposed based on finite difference and finite volume methods, using explicit and implicit schemes of increased order of accuracy in time and space, such as TVD, ENO, etc.

The system of Navier-Stokes equations is used as a basis for improving the mathematical model of the aerodynamic system.

\section{METHODS}

\subsection{System of Navier-Stokes Equations}

Unsteady Navier-Stokes equations for three-dimensional flow of compressible gas in dimensionless form in Cartesian coordinate system $\mathrm{X}=(\mathrm{x}, \mathrm{y}, \mathrm{z})$ have the form

$$
\frac{\partial U}{\partial t}+\frac{\partial}{\partial x}\left(E-E_{\mathrm{V}}\right)+\frac{\partial}{\partial y}\left(F-F_{\mathrm{V}}\right)+\frac{\partial}{\partial z}\left(G-G_{\mathrm{V}}\right)=0,
$$

where functions

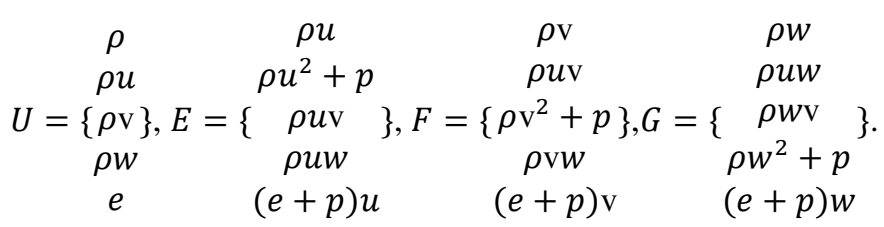

Here, $t$ is time, $[r h o]$ is the density, $(\mathrm{u}, \mathrm{v}, \mathrm{w})$ - components of the velocity vector $\mathrm{V}$, respectively, in directions $(\mathrm{x}$, $\mathrm{y}, \mathrm{z}), p$ - pressure, $e$ - total energy per unit volume of gas, which for an ideal gas can be represented in the form $e=\rho\left(\varepsilon+\frac{u^{2}+v^{2}+w^{2}}{2}\right)$, where $\varepsilon=\frac{1}{\gamma-1} \frac{p}{\rho}-$ the internal energy of a gas, [gamma] - the ratio of heat capacities at constant pressure and volume.

Further,

$$
\begin{aligned}
& 0 \quad 0 \quad 0 \quad d_{x}=u \sigma_{x x}+v \tau_{x y}+w \tau_{x z}+q_{x} \\
& \begin{array}{cccc}
\sigma_{x x} & \tau_{x y} & d_{y}=u \tau_{x y}+\mathrm{v} \sigma_{y y}+w \sigma_{y z}+q_{y}
\end{array}, \\
& E_{\mathrm{v}}=\left\{\tau_{x y}\right\}, F_{\mathrm{v}}=\underset{\tau_{x z}}{\left\{\sigma_{y y}\right\}}, G_{\mathrm{v}}=\underset{\tau_{y z}}{\left\{\tau_{y z}\right\}}, d_{z}=u \tau_{x z}+\mathrm{v} \tau_{y z}+w \sigma_{z z}+q_{z}, \\
& \begin{array}{cccc}
\tau_{x z} & \tau_{y z} & \sigma_{z z} \\
d_{x} & d_{y} & d_{z}
\end{array} \quad \operatorname{divV}=\frac{\partial u}{\partial x}+\frac{\partial v}{\partial y}+\frac{\partial w}{\partial z}, \\
& \sigma_{x x}=\frac{\mu}{\operatorname{Re}}\left(2 \frac{\partial u}{\partial x}-\frac{2}{3} d i v V\right), \sigma_{y y}=\frac{\mu}{\operatorname{Re}}\left(2 \frac{\partial v}{\partial y}-\frac{2}{3} d i v V\right), \sigma_{z z}=\frac{\mu}{\operatorname{Re}}\left(2 \frac{\partial w}{\partial z}-\frac{2}{3} d i v V\right), \\
& \tau_{x y}=\frac{\mu}{\operatorname{Re}}\left(\frac{\partial u}{\partial x}+\frac{\partial \mathrm{v}}{\partial y}\right), \tau_{x z}=\frac{\mu}{\operatorname{Re}}\left(\frac{\partial u}{\partial x}+\frac{\partial \mathrm{w}}{\partial z}\right), \tau_{y z}=\frac{\mu}{\operatorname{Re}}\left(\frac{\partial \mathrm{v}}{\partial y}+\frac{\partial w}{\partial z}\right), \\
& q_{x}=\frac{\gamma}{\gamma-1} \frac{\mu}{\operatorname{Re} \operatorname{Pr}} \frac{\partial \mathrm{T}}{\partial x}, q_{y}=\frac{\gamma}{\gamma-1} \frac{\mu}{\operatorname{Re} \operatorname{Pr}} \frac{\partial \mathrm{T}}{\partial y}, q_{z}=\frac{\gamma}{\gamma-1} \frac{\mu}{\operatorname{Re} \operatorname{Pr}} \frac{\partial \mathrm{T}}{\partial z} .
\end{aligned}
$$

Dimensionless variables are defined through dimensional variables that are marked with a stroke:

$$
t=\sqrt{\frac{p_{0}^{\prime}}{\rho_{0}^{\prime}}} \frac{t^{\prime}}{L^{\prime}}, X=\frac{X^{\prime}}{L^{\prime}}, V=\sqrt{\frac{\rho_{0}^{\prime}}{p_{0}^{\prime}}} V^{\prime}, \rho=\frac{\rho^{\prime}}{\rho_{0}^{\prime}}, p=\frac{p^{\prime}}{p_{0}^{\prime}}, T=\frac{T^{\prime}}{T_{0}^{\prime}}, \mu=\frac{\mu^{\prime}}{\mu_{0}^{\prime}} .
$$


The subscript "zero" means the value of the parameter in the unperturbed stream. Here, L is the characteristic size, $\mathrm{X}=(\mathrm{x}, \mathrm{y}, \mathrm{z})$ and $\mathrm{V}=(\mathrm{u}, \mathrm{v}, \mathrm{w})$.

It is assumed that the Prandtl number $\operatorname{Pr}=\frac{\mu c_{p}}{\lambda}$ is constant, here $c_{p}-$ is the heat capacity coefficient, [lambda] - is the heat conductivity coefficient, the viscosity coefficient [mu] can be calculated by the formula $\mu=T^{k}$, where $0.5 \leq k \leq$ 1.5, $\operatorname{Re}=\sqrt{p_{0}^{\prime} \rho_{0}} L^{\prime} / \mu_{0}^{\prime}$ - is the Reynolds number.

The system of differential equations is supplemented by the equation of state $p=\rho R T$, where $T-$ is the temperature, $R-$ is universal gas constant. In dimensionless form, the equation of state has the form $p=\rho T$. Thus, a complete system of Navier-Stokes equations for perfect gas flows in the absence of external mass forces is given. It is assumed that the heat to the medium can only come as a result of thermal conductivity.

\subsection{System of Navier-Stokes Equations in Generalized Curvilinear Coordinate System}

Using a Cartesian coordinate system to adequately describe flows with complex topology when solving problems by finite difference methods is difficult for two reasons: interpolation procedures are needed to obtain boundary conditions; there are difficulties in describing the computational grid, since the computational grid is not rectangular. So, let us move on to an arbitrary curvilinear coordinate system. The uniform grid for the difference approximation of the initial equations will be set in the following coordinate system:

$$
\tau=t, \xi=\xi(t, x, y, z), \eta=\eta(t, x, y, z), \zeta=\zeta(t, x, y, z)
$$

The use of generalized transformation allows to keep strictly conservative form of equations. The equations take the form

$\frac{\partial}{\partial \tau} \frac{U}{J}+\frac{\partial}{\partial \xi} \frac{\bar{E}}{J}+\frac{\partial}{\partial \eta} \frac{\bar{F}}{J}+\frac{\partial}{\partial \zeta} \frac{\bar{G}}{J}=0$

where

$$
\begin{aligned}
& \bar{E}=\xi_{t} U+\xi_{x}\left(E-E_{\mathrm{v}}\right)+\xi_{y}\left(F-F_{\mathrm{v}}\right)+\xi_{z}\left(G-G_{\mathrm{v}}\right), \\
& \bar{F}=\eta_{t} U+\eta_{x}\left(E-E_{\mathrm{v}}\right)+\eta_{y}\left(F-F_{\mathrm{v}}\right)+\eta_{z}\left(G-G_{\mathrm{v}}\right), \\
& \bar{G}=\zeta_{t} U+\zeta_{x}\left(E-E_{\mathrm{v}}\right)+\zeta_{y}\left(F-F_{\mathrm{v}}\right)+\zeta_{z}\left(G-G_{\mathrm{v}}\right) .
\end{aligned}
$$

The coefficients of the transformation matrix can be calculated by the following formulas:

$$
\begin{aligned}
& \xi_{x}=J\left(\frac{\partial y}{\partial \eta} \frac{\partial z}{\partial \zeta}-\frac{\partial z}{\partial \eta} \frac{\partial y}{\partial \zeta}\right), \xi_{y}=J\left(\frac{\partial z}{\partial \eta} \frac{\partial x}{\partial \zeta}-\frac{\partial x}{\partial \eta} \frac{\partial z}{\partial \zeta}\right), \xi_{z}=J\left(\frac{\partial x}{\partial \eta} \frac{\partial y}{\partial \zeta}-\frac{\partial y}{\partial \eta} \frac{\partial x}{\partial \zeta}\right), \\
& \eta_{x}=J\left(\frac{\partial z}{\partial \xi} \frac{\partial y}{\partial \zeta}-\frac{\partial y}{\partial \xi} \frac{\partial z}{\partial \zeta}\right), \eta_{y}=J\left(\frac{\partial z}{\partial \xi} \frac{\partial x}{\partial \zeta}-\frac{\partial x}{\partial \xi} \frac{\partial z}{\partial \zeta}\right), \eta_{z}=J\left(\frac{\partial x}{\partial \xi} \frac{\partial y}{\partial \zeta}-\frac{\partial y}{\partial \xi} \frac{\partial x}{\partial \zeta}\right), \\
& \zeta_{x}=J\left(\frac{\partial z}{\partial \xi} \frac{\partial y}{\partial \eta}-\frac{\partial y}{\partial \xi} \frac{\partial z}{\partial \eta}\right), \zeta_{y}=J\left(\frac{\partial z}{\partial \xi} \frac{\partial x}{\partial \eta}-\frac{\partial x}{\partial \xi} \frac{\partial z}{\partial \eta}\right), \zeta_{z}=J\left(\frac{\partial x}{\partial \xi} \frac{\partial y}{\partial \eta}-\frac{\partial y}{\partial \xi} \frac{\partial x}{\partial \eta}\right),
\end{aligned}
$$

where $J-$ is the Jacobian of the transformation, which is defined by the formula

$J^{-1}=\frac{\partial x}{\partial \xi} \frac{\partial y}{\partial \eta} \frac{\partial z}{\partial \zeta}+\frac{\partial x}{\partial \zeta} \frac{\partial y}{\partial \xi} \frac{\partial z}{\partial \eta}+\frac{\partial x}{\partial \eta} \frac{\partial y}{\partial \zeta} \frac{\partial z}{\partial \xi}-\frac{\partial x}{\partial \xi} \frac{\partial y}{\partial \zeta} \frac{\partial z}{\partial \eta}-\frac{\partial x}{\partial \zeta} \frac{\partial y}{\partial \eta} \frac{\partial z}{\partial \xi}-\frac{\partial x}{\partial \eta} \frac{\partial y}{\partial \xi} \frac{\partial z}{\partial \zeta}$.

The use of generalized transformation allows to construct a uniform grid in the form of a unit cube. The 
coefficients of the transformation matrix can be calculated either analytically (if the transformation is given algebraically) or using difference formulae according to equations (if the distribution of nodes in the physical domain of the calculation is given). The construction of a grid in a physical domain adapted to real geometry is currently an intensively developed problem of computational mathematics. Suppose that the grid is constructed so that near the surface of the body, the coordinate lines $([\mathrm{xi}]=$ const . $[$ zeta $]=$ const . $)$ are oriented along the normal to the surface $[$ eta $]=$ const .

When writing equations, it is assumed that the derivatives that are present in the expressions for $\mathbf{E v}, \mathbf{F v}$ and $\mathbf{G v}$ are transformed according to the rules of differentiation of complex functions. These components are responsible for the presence of viscous forces. A wide class is represented by flows in which the viscosity forces significantly affect the flow only in thin layers or near the surface of the streamlined body. In the case of weak visco-inviscid interaction (there is no interaction of the boundary or shear layer with the incident jumps of compaction and separation of the flow). It is possible to solve the problem by the classical approach, which consists of dividing the problem into inviscid and viscous.

\subsection{System of Euler Equations}

The system of Euler equations in the generalized coordinate system is obtained from the above system by the limit transition. Viscous terms are assumed to be zero. The system of Euler equations has the form

$$
\begin{aligned}
& \frac{\partial}{\partial \tau} \frac{U}{J}+\frac{\partial}{\partial \xi} \frac{\hat{E}}{J}+\frac{\partial}{\partial \eta} \frac{\hat{F}}{J}+\frac{\partial}{\partial \zeta} \frac{\hat{G}}{J}=0, \\
& \text { where } \\
& \hat{E}=\xi_{t} U+\xi_{x} E+\xi_{y} F+\xi_{z} G, \\
& \hat{F}=\eta_{t} U+\eta_{x} E+\eta_{y} F+\eta_{z} G, \\
& \hat{G}=\zeta_{t} U+\zeta_{x} E+\zeta_{y} F+\zeta_{z} G .
\end{aligned}
$$

If we enter the contravariant components of the velocity vector

$$
\hat{u}=\xi_{t}+\xi_{x} u+\xi_{y} \mathrm{v}+\xi_{z} w, \mathrm{v}^{\wedge}=\eta_{t}+\eta_{x} u+\eta_{y} \mathrm{v}+\eta_{z} w, \widehat{w}=\zeta_{t}+\zeta_{x} u+\zeta_{y} \mathrm{v}+\zeta_{z} w
$$

the vectors $\hat{\mathbf{E}}, \hat{\mathbf{F}}, \hat{\mathbf{G}}$ can be represented as

$$
\begin{array}{ccc}
\rho \hat{u} & \rho \mathrm{v}^{\wedge} & \rho \widehat{w} \\
\rho u \hat{u}+\xi_{x} p & \rho u \mathrm{v}^{\wedge}+\eta_{x} p & \rho u \widehat{w}+\zeta_{x} p \\
\hat{E}=\left\{\rho \mathrm{v} \hat{u}+\xi_{y} p\right\}, \hat{F}=\left\{\rho \mathrm{v}^{\wedge}+\eta_{y} p\right\}, \widehat{G}=\left\{\rho \mathrm{v} \widehat{w}+\zeta_{y} p\right\} . \\
\rho \mathrm{w} \hat{u}+\xi_{y} p & \rho \mathrm{w} \mathrm{v}^{\wedge}+\eta_{y} p & \rho \mathrm{w} \widehat{w}+\zeta_{y} p \\
(e+p) \hat{u} & (e+p) \mathrm{v}^{\wedge} & (e+p) \widehat{w}
\end{array}
$$

\subsection{System of Navier-Stokes Equations in the Thin Layer Approximation}

For the calculation of flows with strong viscous-inviscid interaction is considered a single equation that takes account of both inviscid and viscous forces. It is assumed that the dissipative processes in the direction of the normal to the surface of the body or shear layer are much more dissipative processes in tangent directions. Hence, in the Navier-Stokes equations, when calculating dissipative terms, it is possible to neglect terms with derivatives in tangent directions. As a result, we obtain equations that contain all the terms of the Euler equations, the boundary layer equations, and the second derivative in the normal-to-surface momentum equation. The resulting system of equations will later be called the Navier-Stokes 
system of equations in the thin layer approximation. This system has the form

$$
\frac{\partial}{\partial \tau} \frac{U}{J}+\frac{\partial}{\partial \xi} \frac{\hat{E}}{J}+\frac{\partial}{\partial \eta} \frac{\hat{F}}{J}+\frac{\partial}{\partial \zeta} \frac{\hat{G}}{J}=\frac{\partial}{\partial \eta} \frac{S}{J},
$$

where

$$
\begin{aligned}
& m_{1} \frac{\partial u}{\partial \eta}+m_{2} \eta_{x} \quad m_{1}=\eta_{x}^{2}+\eta_{y}^{2}+\eta_{z}^{2}, \\
& S=\frac{\mu}{\operatorname{Re}}\left\{m_{1} \frac{\partial \mathrm{v}}{\partial \eta}+m_{2} \eta_{y}\right\}, \quad m_{2}=\frac{1}{3}\left[\eta_{x} \frac{\partial u}{\partial \eta}+\eta_{y} \frac{\partial \mathrm{v}}{\partial \eta}+\eta_{z} \frac{\partial w}{\partial \eta}\right], \\
& m_{1} \frac{\partial \mathrm{w}}{\partial \eta}+m_{2} \eta_{z} \quad m_{3}=m_{1}\left[\frac{\gamma}{\gamma-1} \frac{1}{\operatorname{Pr}} \frac{\partial T}{\partial \eta}+\frac{\partial}{\partial \eta} \frac{u^{2}+\mathrm{v}^{2}+w^{2}}{2}\right]+m_{2}\left[\eta_{x} u+\eta_{y} \mathrm{v}+\eta_{z} w\right],
\end{aligned}
$$

$\eta=$ const. sets the boundary - the surface of the body.

Formally, the Navier-Stokes equations in the "thin" layer approximation are obtained by estimating the derivatives $\partial / \partial \xi$ (tangent) and $\partial / \partial \eta$ (normal) in the viscous terms and excluding all derivatives $\partial / \partial \xi$.

When solving problems of aerodynamics in many important cases, it is necessary to take into account the effects of viscosity. The separation of the flow and the formation of vortex structures, the interaction of shock waves with the boundary layer fundamentally changes the flow structure, which, in turn, determines the pressure distribution and the total aerodynamic properties. In many modern aircrafts, vortex structures are used purposefully to achieve certain aerodynamic properties in a wide range of angles of attack and deflection of the control of aerodynamic surfaces. For this reason, it is often not possible to make a classical partition of the problem into inviscid (within the Euler equations) for determining the pressure distribution and viscous (within the boundary layer equations) for estimating friction. It is necessary to use more complex mathematical models. However, when solving three-dimensional problems of external flow at high temperatures, the possibilities of real computer technology are limited to the condensation of nodes only in one coordinate direction. The greatest gradients due to viscosity are realized in a thin viscous layer near the surface of the body. Accordingly, the thickening of the nodes is carried out in the direction of the normal to the surface of the body, which makes it possible to calculate the terms of the boundary layer equations. For this reason, from the point of view of real computational possibilities in calculating the dissipative term of the Navier-Stokes equations, it seems reasonable to limit the calculation of the terms included in the boundary layer equations, namely only the second derivatives of the normal to the surface of the body.

This model is called the Navier-Stokes equations in the thin layer approximation. This model should be distinguished from the thin shock layer model, which is used to solve hypersonic aerodynamics problems near blunted bodies.

The system of Navier-Stokes equations in the thin layer approximation contains all the terms of the Euler and boundary layer equations, which allows to take into account both the inviscid and the main viscous effects, and as a result, the visco-inviscid interaction within the framework of unified equations. The use of this model is mainly due to the resources of existing computer calculation.

\subsection{Turbulence Models}

The calculation of turbulent flows is carried out in the framework of averaged Navier-Stokes equations with different closure models: algebraic model from (Baldwin and Lomax, 1978), model from (Tulapurkara, 1997; Belov and Isaev, 
2001), model of time-averaged kinetic energy of turbulent motion and turbulent viscosity, LARGE-scale Les vortices, direct numerical simulation (DNS).

\subsection{Numerical Method}

We briefly describe an explicit second-order difference scheme of predictor-corrector approximation (see MacCormack, 1969). We introduce a grid with step sizes $h_{1}, h_{2}$ and $h_{3}$, such as that of $\xi_{m}=m h_{1}, \eta_{k}=k h_{2}$ and $\zeta_{l}=l h_{3}$. Integration in time to achieve a stationary state is carried out on the basis of a two-step scheme, which is formally represented as

$$
\begin{aligned}
& \mathbf{U}_{m, k, l}^{(1)}=\mathbf{U}^{n}-\frac{\tau}{h_{1}}\left(\mathbf{E}_{m}^{n}-\mathbf{E}_{m-1}^{n}\right)-\frac{\tau}{h_{2}}\left(\mathbf{F}_{k}^{n}-\mathbf{F}_{k-1}^{n}\right)-\frac{\tau}{h_{3}}\left(\mathbf{G}_{l}^{n}-\mathbf{G}_{l-1}^{n}\right), \\
& \mathbf{U}_{m, k, l}^{n+1}=\frac{1}{2}\left\{\mathbf{U}^{n}+\mathbf{U}^{(1)}-\frac{\tau}{h_{1}}\left(\mathbf{E}_{m+1}^{(1)}-\mathbf{E}_{m}^{(1)}\right)-\frac{\tau}{h_{2}}\left(\mathbf{F}_{k+1}^{(1)}-\mathbf{F}_{k}^{(1)}\right)-\frac{\tau}{h_{3}}\left(\mathbf{G}_{l+1}^{(1)}-\mathbf{G}_{l}^{(1)}\right)\right\} .
\end{aligned}
$$

Here, the superscript (1) denotes that the function values are calculated for intermediate solution values $\mathbf{U}^{(1)}$. In addition to the written difference relations, the next final step is the "smoothing" of the solution, often necessary in areas with shock waves.

In fact, convective terms are represented by one-sided differences with a change of direction, and dissipative terms are represented by symmetric differences.

When using explicit difference schemes, the time step size is limited by the stability condition of the calculation; we use the method of local linearization.

In the case of compressible gas flows, the Euler equation system is written as

$$
\frac{\partial}{\partial \tau} \frac{\mathbf{U}}{J}+\mathbf{A} \frac{\partial \mathbf{U}}{\partial \xi}+\mathbf{B} \frac{\partial \mathbf{U}}{\partial \eta}+\mathbf{C} \frac{\partial \mathbf{U}}{\partial \zeta}=0
$$

Eigenvalues of a matrix $\mathbf{A}, \mathbf{B}$ and $\mathbf{C}$ :

$$
\begin{array}{ll}
\sigma_{1,2,3}^{A}=A=\xi_{x} u+\xi_{y} \mathrm{v}+\xi_{\mathrm{z}} w, & \sigma_{4,5}^{A}=A \pm \sqrt{\gamma \frac{p}{\rho}\left(\xi_{x}^{2}+\eta_{y}^{2}+\zeta_{z}^{2}\right)}, \\
\sigma_{1,2,3}^{B}=B=\eta_{x} u+\eta_{y} \mathrm{v}+\eta_{z} w, & \sigma_{4,5}^{B}=B \pm \sqrt{\gamma \frac{p}{\rho}\left(\eta_{x}^{2}+\eta_{y}^{2}+\eta_{z}^{2}\right)}, \\
\sigma_{1,2,3}^{C}=C=\zeta_{x} u+\zeta_{y} \mathrm{v}+\zeta_{\mathrm{z}} w, & \sigma_{4,5}^{C}=C \pm \sqrt{\gamma \frac{p}{\rho}\left(\zeta_{x}^{2}+\zeta_{y}^{2}+\zeta_{z}^{2}\right)} .
\end{array}
$$

The restriction on the time step is represented in the form

$$
\Delta t \leq \mathrm{Ku} \frac{\Delta \xi}{\max \left|\sigma^{\mathrm{A}}\right|}, \Delta t \leq \mathrm{Ku} \frac{\Delta \eta}{\max \left|\sigma^{\mathrm{B}}\right|}, \Delta t \leq \mathrm{Ku} \frac{\Delta \zeta}{\max \left|\sigma^{\mathrm{C}}\right|},
$$

where $\mathrm{Ku}$ is the Courant number. 
Viscous dissipative effects have a great influence on the flow near the surface of the body. In addition, actually used computational grids due to limited computational resources do not provide the possibility of sufficient thickening of nodes at a distance from the body. For these reasons, the dissipative term can be taken into account, and therefore the calculation can be carried out only in the region, where $0 \leq \eta \leq \bar{\eta}$ and $\bar{\eta}<1$ ( $\eta=1-$ the outer boundary), and $\bar{\eta}$ determined either experimentally, or a condition must be set for its determination. In the region $\bar{\eta} \leq \eta \leq 1$, the dissipative term is small and can be put equal to zero.

The introduction of the dissipative term changes the stability condition. The condition of the restriction on the step, taking into account the dissipative term has the form:

$$
\Delta t \leq \mathrm{Ku} \frac{\Delta \eta}{\left|\sigma^{\mathrm{B}}\right|+2\left|\sigma^{\mathrm{V}}\right| / \Delta \eta}, \text { где } \sigma^{V}=\left(\eta_{x}^{2}+\eta_{y}^{2}+\eta_{z}^{2}\right) \frac{\gamma \mu}{\rho \operatorname{Re} \operatorname{Pr}} .
$$

Note that accounting for viscosity in the complete or simplified Navier-Stokes equations requires a strong thickening of nodes in viscous thin layers, which leads to a significant reduction in the integration step over time, but this limitation is mainly due to the rate of propagation of perturbations, not viscosity. The type of the step restriction taking into account the dissipative term reveals that with a fixed grid, an increase in Re leads to the possibility of increasing the integration step. If the Re decreases (i.e., the viscosity of the flow increases), the possible integration step decreases. In reality, the grid step is related to the Reynolds number. The greater the Re, the greater must be the thickening of the nodes to describe thin viscous layers, and the reduction of Re allows for the use of grids with less thickening. In fact, the design grid should be built with Re in mind.

To perform the calculation, the Courant number is usually assumed to be $\mathrm{Ku}$ from 0.5 to 0.9 . Due to the need to construct a substantially uneven grid, the integration step in time is determined in the region of the greatest condensation of nodes. Establishing a single integration step is extremely slow. A local integration step is used to speed up the installation: the integration step at each node is selected locally based on local conditions. This ensures that the distribution of the disturbance with a speed of one knot for one integration step with $\mathrm{Ku}=1.0$. Using a local integration step leads to the need to reduce the number of chimes from 0.1 to 0.25 . The use of the local integration step does not allow to consider nonstationary problems, but if only the steady-state flow is of interest, then the local step allows to significantly reduce the total number of integration steps before the solution is established.

The explicit scheme described has second-order accuracy in time and spatial variables on smooth solutions. Changing the pattern or increasing the number of iterative steps in time can increase the order of approximation in space or time.

The scheme of the second order in accuracy in time and the fourth in space for a one-dimensional unsteady equation has the form

$$
\begin{aligned}
& U_{m}^{(1)}=U^{n}-\frac{\tau}{6 h_{1}}\left[7\left(E_{m+1}^{n}-E_{m}^{n}\right)-\left(E_{m+2}^{n}-E_{m+1}^{n}\right)\right], \\
& U_{m}^{(1)}=\frac{1}{2}\left\{U^{n}+U^{(1)}-\frac{\tau}{6 h_{1}}\left[7\left(E_{m}^{(1)}-E_{m-1}^{(1)}\right)-\left(E_{m-1}^{(1)}-E_{m-2}^{(1)}\right)\right]\right\} .
\end{aligned}
$$

Increasing the order of approximation in time is achieved by introducing additional time steps (Runge-Kutta type schemes): 


$$
\begin{aligned}
& U_{m}^{(1)}=U^{n}-\frac{1}{2} \frac{\tau}{h_{1}}\left(E_{m}^{n}-E_{m-1}^{n}\right), \\
& U_{m}^{(2)}=U^{n}-\frac{1}{2} \frac{\tau}{h_{1}}\left(E_{m+1}^{(1)}-E_{m}^{(1)}\right) \\
& U_{m}^{(3)}=U^{n}-\frac{1}{2} \frac{\tau}{h_{1}}\left(E_{m}^{(2)}-E_{m-1}^{(2)}\right), \\
& U_{m}^{n+1}=U^{n}-\frac{1}{6} \frac{\tau}{h_{1}}\left\{\left(E_{m}^{n}-E_{m-1}^{n}\right)+2\left(E_{m+1}^{(1)}-E_{m}^{(1)}\right)+2\left(E_{m}^{(2)}-E_{m-1}^{(2)}\right)+\left(E_{m+1}^{(3)}-E_{m}^{(3)}\right)\right\}
\end{aligned}
$$

The use of explicit schemes allows a simple implementation of a numerical algorithm for solving differential equations on multiprocessor computer technology.

\subsection{The Process of Designing a Model and Creating a Computational Grid}

To solve the flow problem, it is necessary to describe the geometry of the object of study. The construction of the geometry depends on the initial information and the objectives of the study. At all stages of design, the choice of a real form is one of the central tasks. There are a number of computer applications that allow you to automate geometric design. An important feature of these systems is the ability to directly observe the results of the work on the computer screen. In the construction of complex geometry, various methods are used: "solid-state" method, analytical description and spline theory. Since each system has its own goals and uses original methods of describing geometry, the transfer of information from one system to another is difficult. For data exchange between automation systems, special data formats have been developed, focusing on which and having the means of their analysis, it is possible to obtain the necessary geometry data (for example, data on the surface of a streamlined body, perpendicular to its surface).

Solid-state geometric modeling system consists of a set of tools that allow you to interactively create from simple geometric shapes (plane, cone, cylinder, ball, ellipsoid, etc.). The geometry of the real form using the operations of union, intersection and subtraction. The division of geometry into bodies of simple form and the subsequent construction of geometry consists of determining the geometric parameters of simple bodies, determining their position in space and then combining. Such applications of programs for work with geometrical models allow to design and create objects independently, using the saved-up experience from a database, to make geometry of aerodynamic profiles, using sets of various data (for example, radius of curves, angles of inclination of various axes, radii of a fuselage, angle of attack of a wing, degree of placement of a tail part, radii of sides, etc.) from volume primitives. Computer-aided design technology based on the features of models (figure 1), makes it possible to increase the speed of design, improve the accuracy of the values of the studied processes as well as improve the aerodynamic characteristics of the designed airframe, which will largely reduce the complexity, material consumption, error in the design and facilitate the creation of a new aircraft with an optimized shape.

Also, in the development of design, offices and research institutes used various methods of creating models based on three-dimensional scanning of real samples (figure 2). However, such models have a large number of known disadvantages - time-consuming surface preparation, the presence of insensitive areas for the scanner, the complexity of processing a large number of roughness on the surface, etc. 


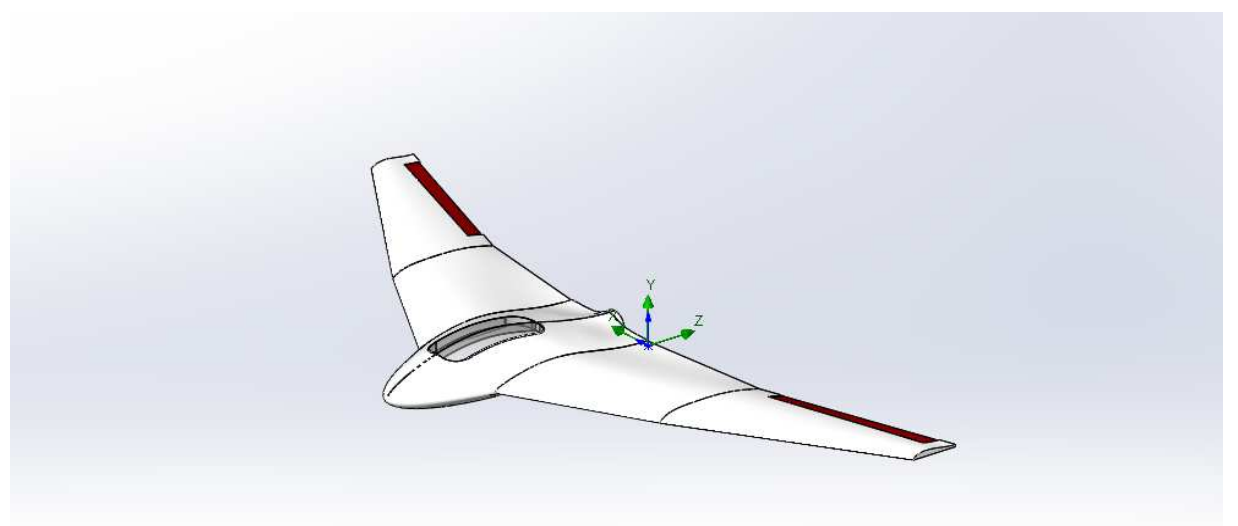

Figure 1: UAV Model Built using Solid-State Design.

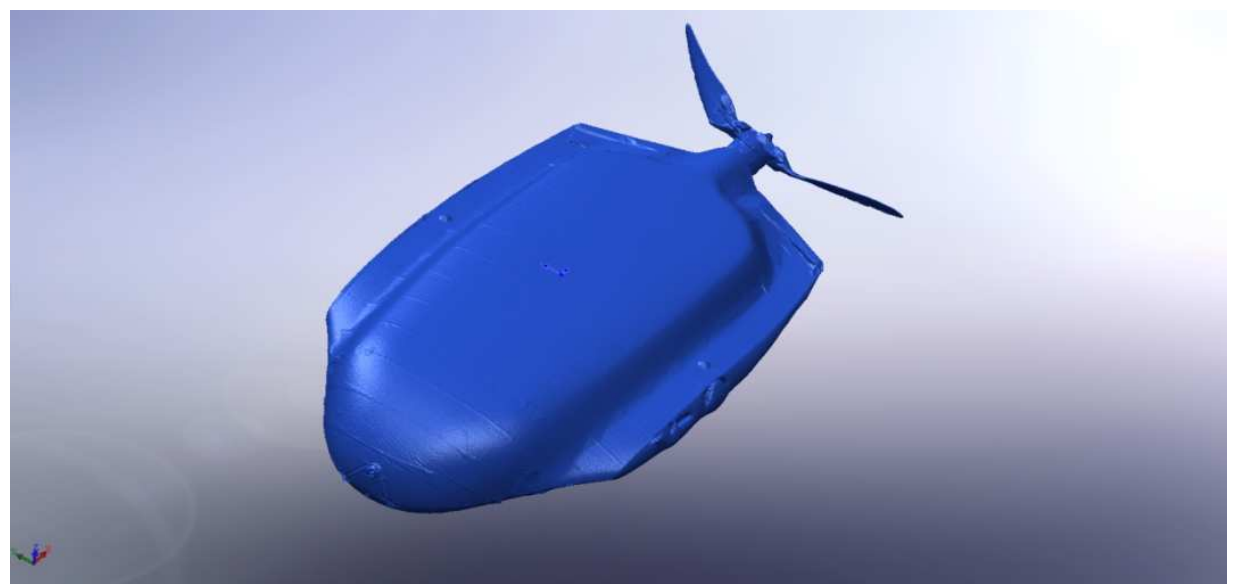

Figure 2: UAV Model, Built using Three-Dimensional Scanning (Revealed Only the Fuselage).

As can be seen from the figures, the most acceptable model for calculations is precisely built because of its symmetry and better streamlining.

When solving flow problems numerically, it is important to construct not only the geometry of the body, but also calculation of grid near it. In order to include grid construction in the design system, you need to "correctly" construct the geometry using the computer-aided design system. Building a grid around arbitrary bodies is a time-consuming process that depends on many factors arising in the course of the project. Quite often such surfaces are given from the point of view of parameterization unsuccessfully. Therefore, not only must the construction of the grid be a sustainable process, but the construction method should not depend on the method of parameterization. For optimization problems, design parameters must be selected, so that they are sensitive to grid changes.

When solving problems of external flow by methods of computational aerodynamics, the calculated grid must be connected to the surface of the streamlined body, which is due to the nature of the flow parallel to the surface of the body. In addition, when taking into account the viscosity to describe the solution, it is necessary to significantly thicken the nodes to the surface of the body to describe the profile of gas-dynamic functions in the thin layer near the body. If these features are not taken into account, it seems impossible to build a solution that is adequate to reality. However, in the external flow region, it is advisable to use a more regular computational grid that is not connected to the surface of the streamlined body. We develop methods of constructing meshes to allow to some extent to smooth out the effects of fractures of the body 
surface to the mesh when removed from it, but the problems associated primarily with the complexity of the topology of the considered task, leads to the need to build a multiblock mesh, sew on some borders. This complicates the algorithms for solving problems. It is proposed to build computational grids near a streamlined body (or several bodies) and in an external flow independently. In addition, the grid near the streamlined body is built only in some neighborhoods of the body, which greatly simplifies its construction.

The calculated main grid (figure 3) is a grid with rectangular cells and is designed to calculate the external inviscid flow.

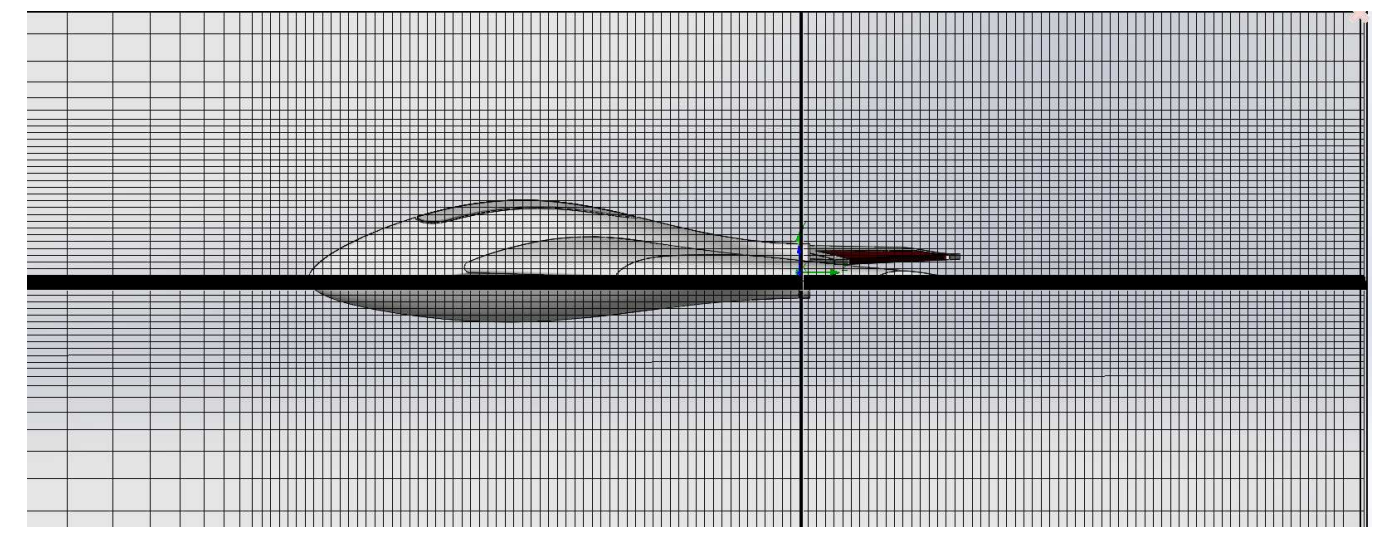

Figure 3: Construction of the Calculated Grid around the Body Under Study.

Thus, during the simulation, we choose a high-speed coordinate system, which is tied to the airspeed of the aircraft. In this case, the speed of the vessel is defined as a vector consisting of components $\mathrm{Vx}, \mathrm{Vy}, \mathrm{Vz}$ : $\mathbf{V}=V_{x} \mathbf{i}+V_{y} \mathbf{j}+V_{z} \mathbf{k}$, and it gives us direct information about the strength of the frontal resistance and lifting force.

For the calculation, we will take the following parameters of the external environment according to the model of the standard atmosphere (Altitude $4000 \mathrm{~m}$ ) (Anderson, 2016):

Medium type: Air

Flight height: $4000 \mathrm{~m}$

Atmospheric pressure at this altitude: $61300 \mathrm{~Pa}$

Temperature: $-100^{\circ} \mathrm{C}$

Center of mass speed: $20 \mathrm{~m} / \mathrm{s}$

Angle of attack: $[$ alpha $]=6$ degrees

The following projects were selected as the models under consideration (figures 4-9):

- The basic model without changes.

- A basic airframe with a single spherical dome fairing mounted at the front of the fuselage.

- Basic airframe with a single spherical dome fairing mounted in the middle of the fuselage.

- Basic airframe with two spherical dome fairings of different diameters, mounted on the front and middle of the fuselage. 
- $\quad$ Base glider with combined domes of different diameters.

- The basic airframe with an elongated fairing of elongated shapes.

As output parameters, we will compare changes in resistance forces from air flows, lift, changes in flow temperature (minimum, average and maximum) as well as moments of forces acting on the airframe.

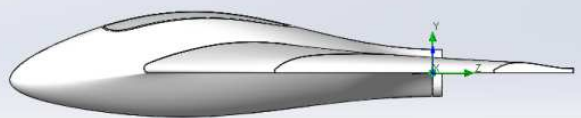

Figure 4: UAV Profile of Project 1.

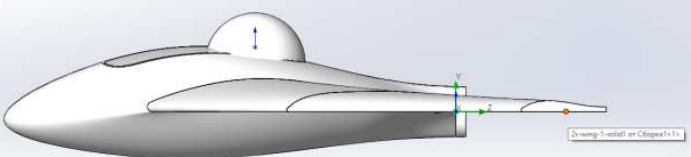

Figure 6: UAV Profile of Project 3.

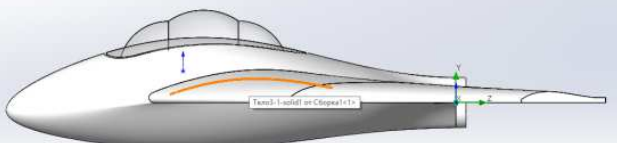

Figure 8: UAV Profile of Project 5.

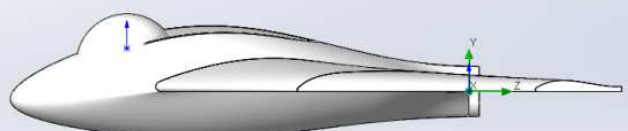

Figure 5: UAV Profile of Project 2.

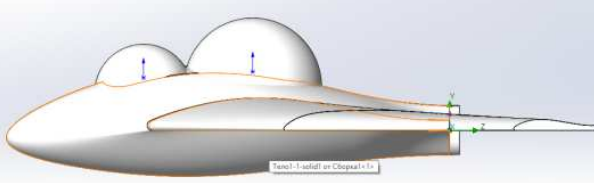

Figure 7: UAV Profile of Project 4.

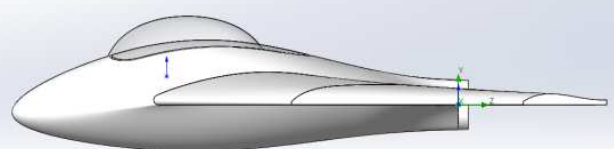

Figure 9: UAV Profile of Project 6.

After that, a mathematical calculation was carried out according to the mathematical model described above. Based on the results of calculations to visualize changes in air flows and pressure redistribution, pressure distribution patterns were constructed in the plane passing through the central axis of symmetry of the airframe (figures 10-15), which allow to see the result of changes in aerodynamic shape. 


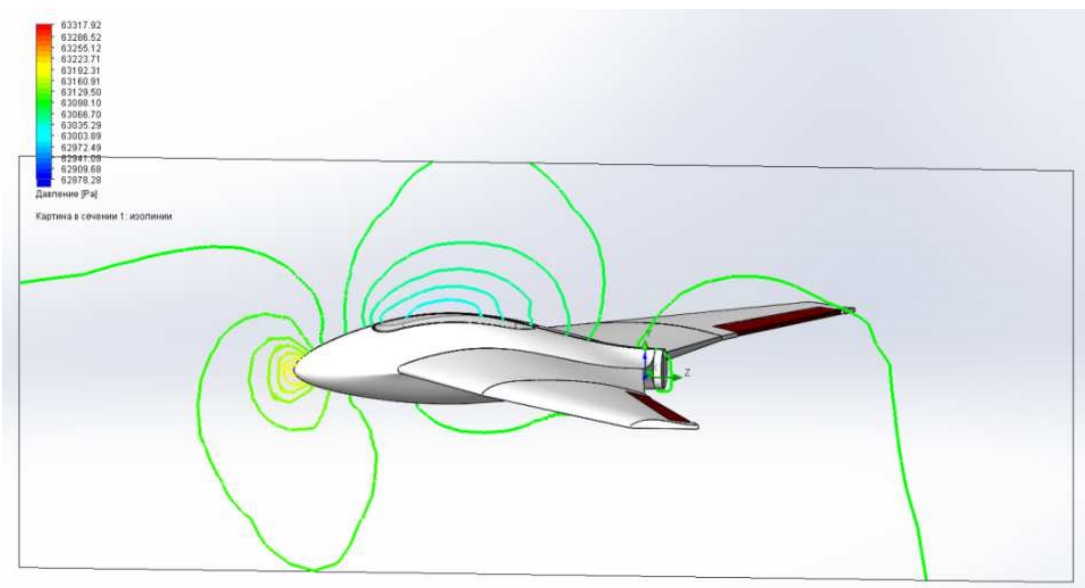

Figure 10: Pressure Isobars in the Symmetry Plane of Project 1.

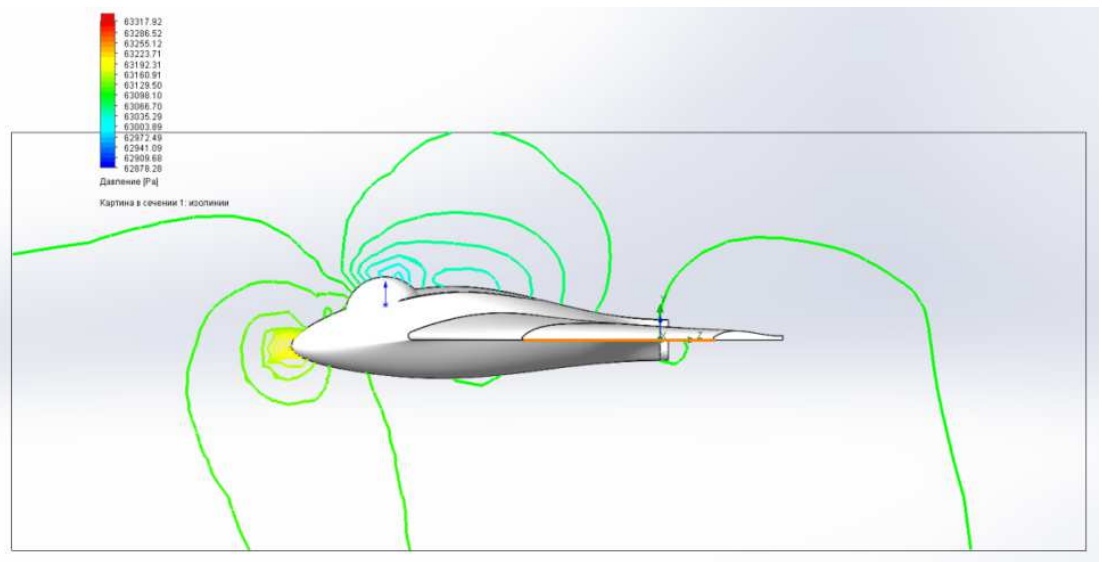

Figure 11: Pressure Isobars in the Symmetry Plane of Project 2.

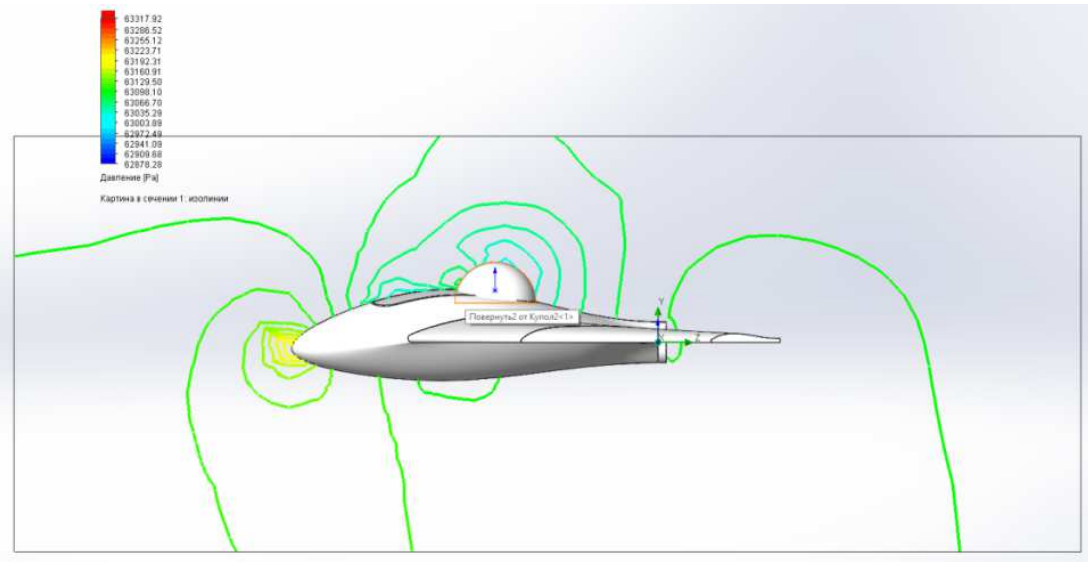

Figure 12: Pressure Isobars in the Symmetry Plane of Project 3. 


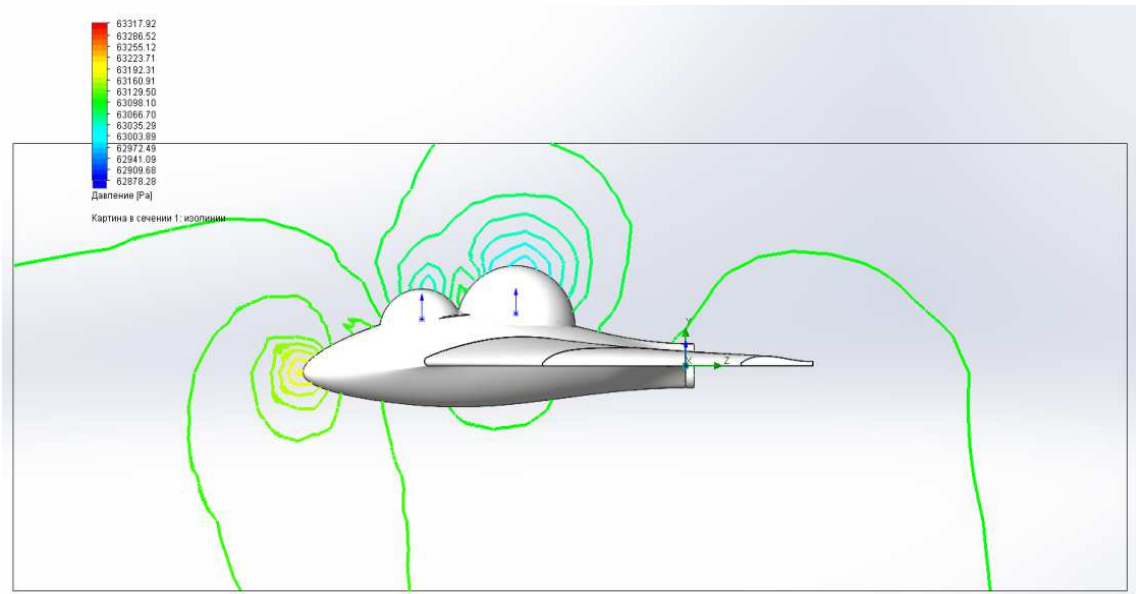

Figure 13: Pressure Isobars in the Symmetry Plane of Project 4.

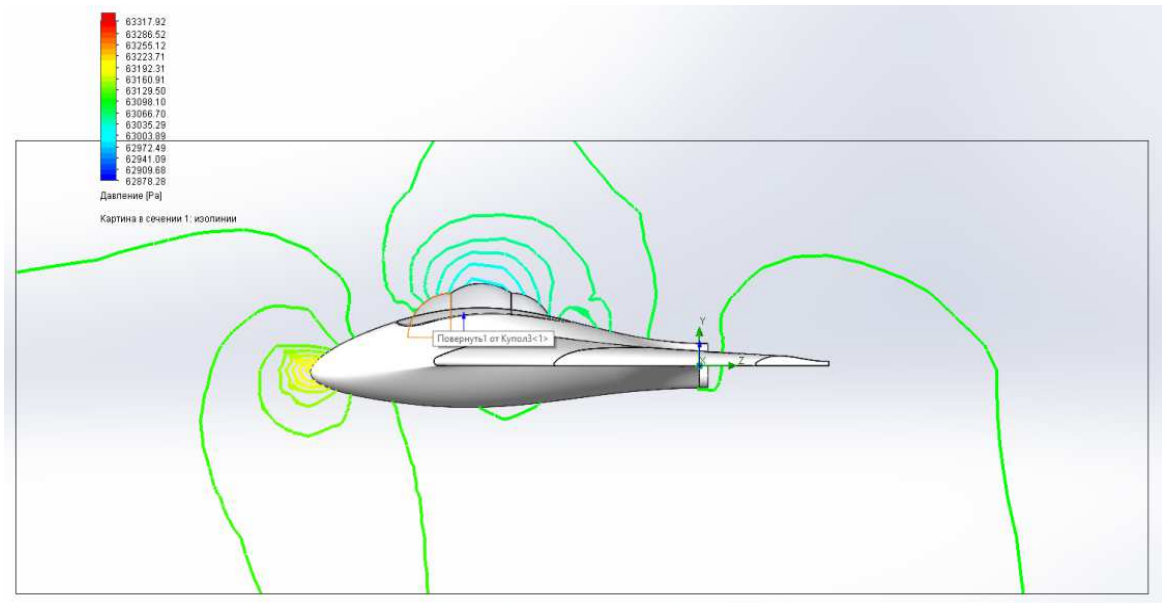

Figure 14: Pressure Isobars in the Symmetry Plane of Project 5

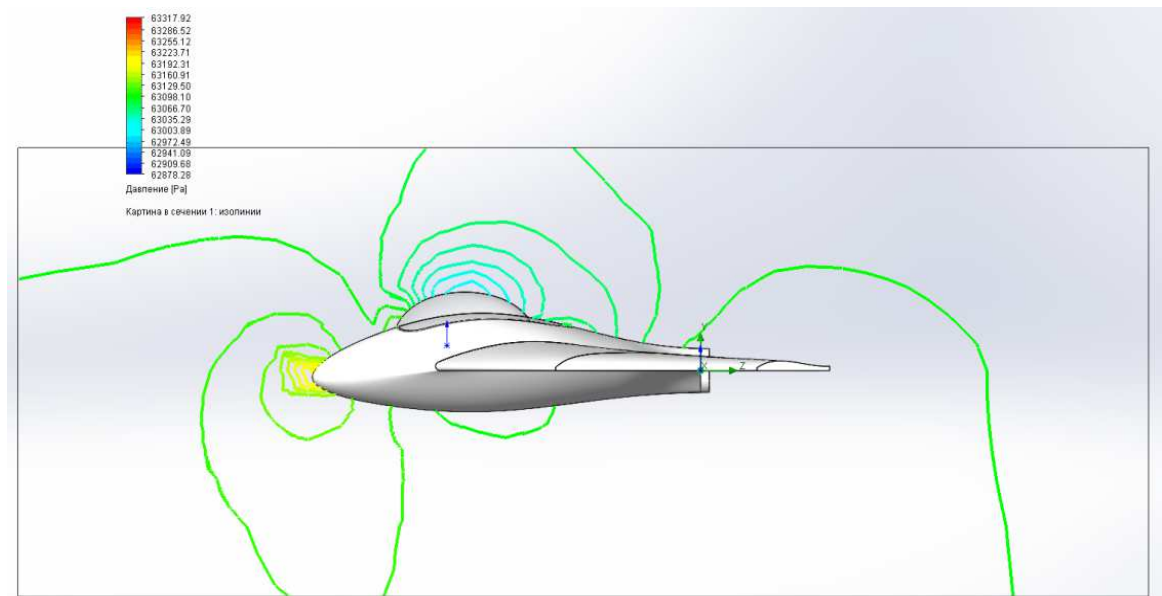

Figure 15: Pressure Isobars in the Symmetry Plane of Project 6.

\section{RESEARCH RESULT}

A detailed analysis of the pressure changes revealed that, indeed, changes in the upper fuselage did not have a significant change in the pressure distribution in the lower airframe. However, there are significant changes in the upper hemisphere 

Mathematical Model of Numerical Simulation

of the surrounding space. The reason for this change lies in the change in the current lines flowing around the airframe. To do this, we also constructed the trajectories of the current lines of the air particles depending on their speed and location.

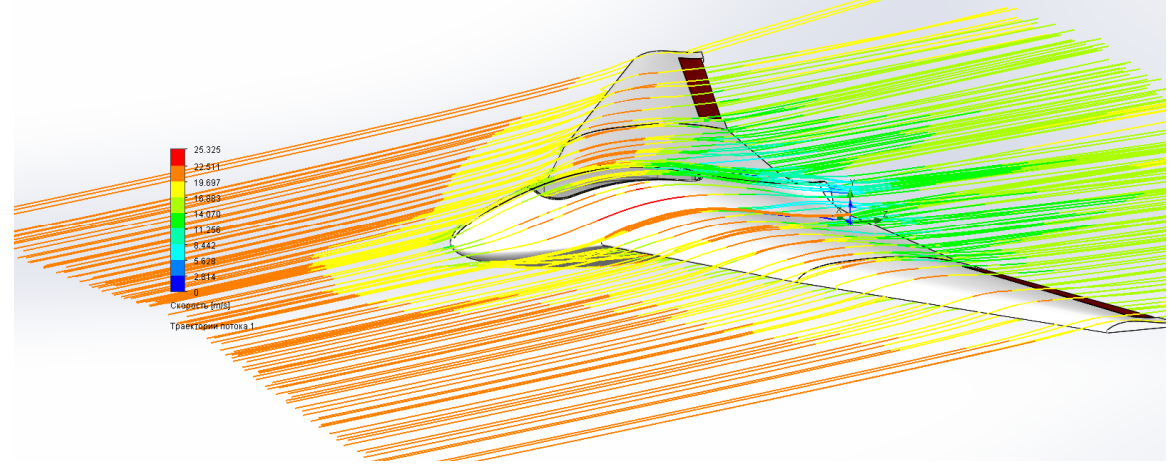

Figure 16: Velocity Distribution of Air Current Lines of Project 1.

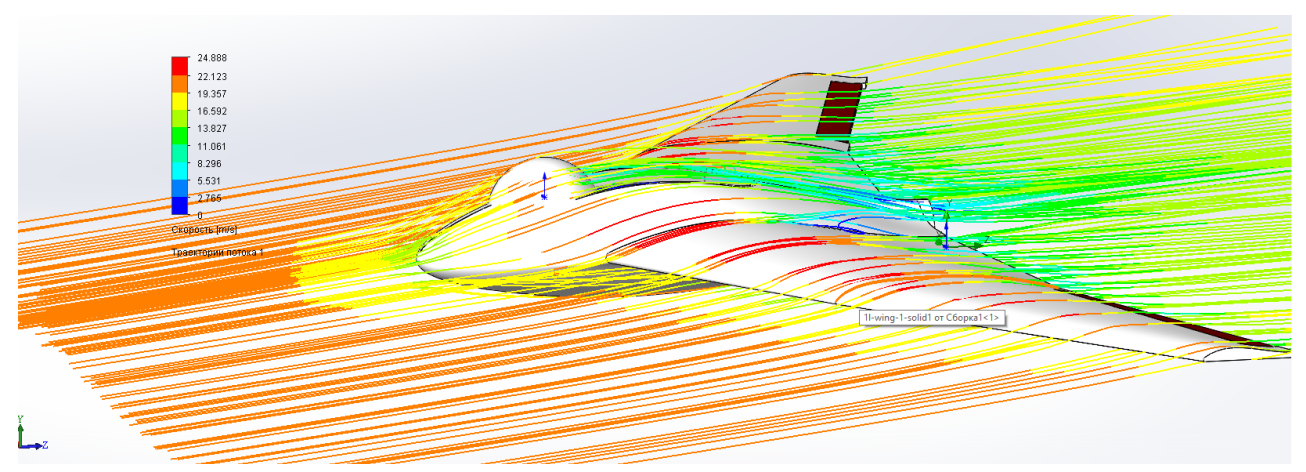

Figure 17: Velocity Distribution of Air Current Lines of Project 2.

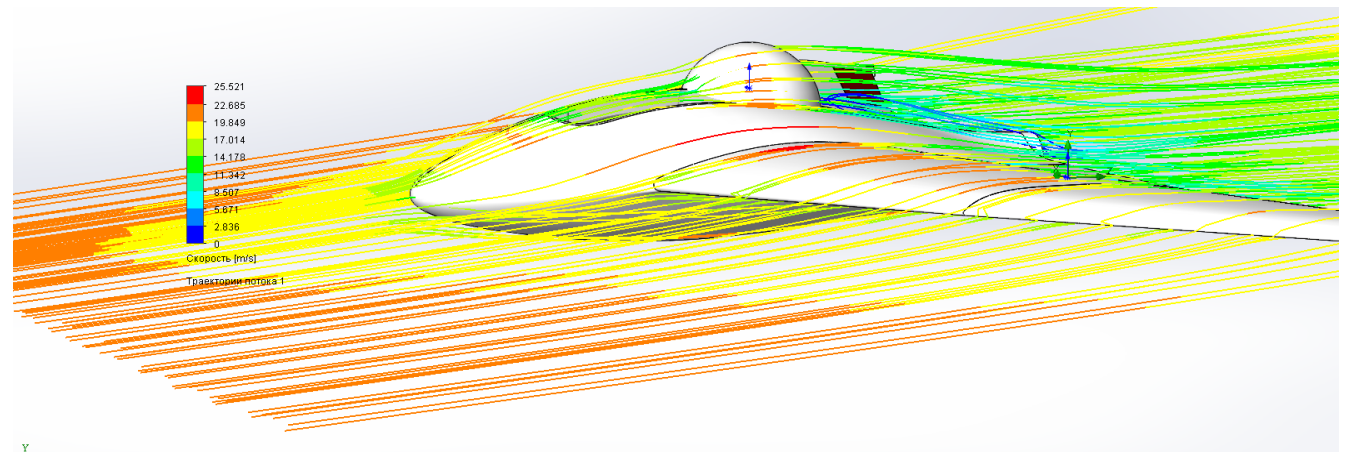

$\stackrel{4}{\longrightarrow}$

Figure 18: Velocity Distribution of Air Current Lines of Project 3.

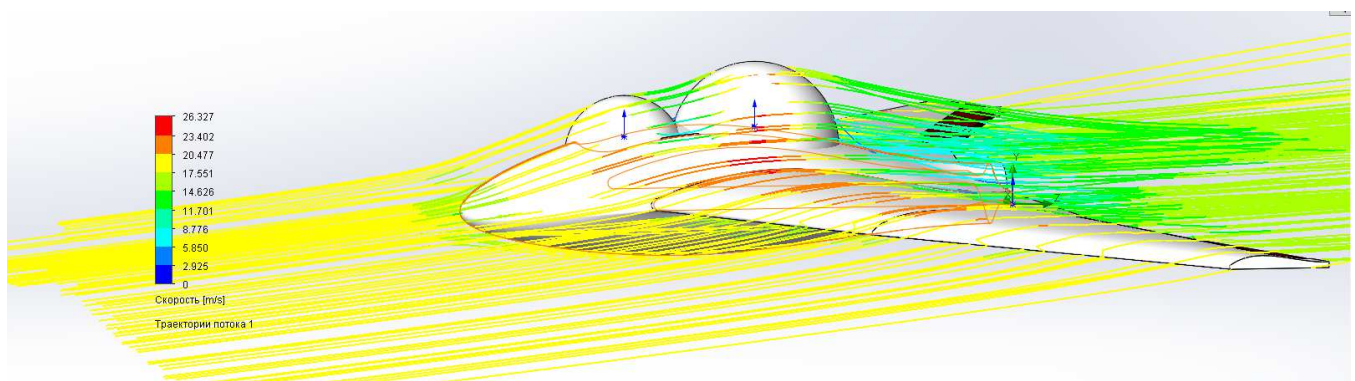

Figure 19: Velocity Distribution of Air Current Lines of Project 4. 


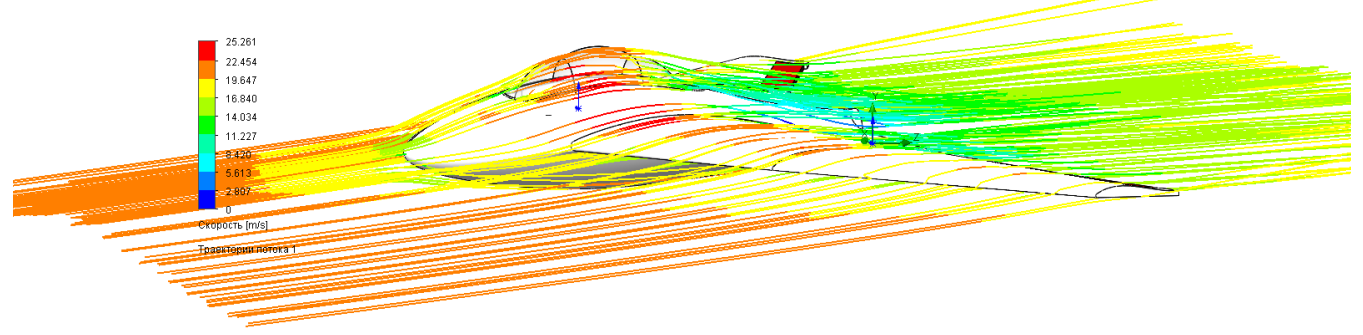

Figure 20: Velocity Distribution of Air Current Lines of Project 5

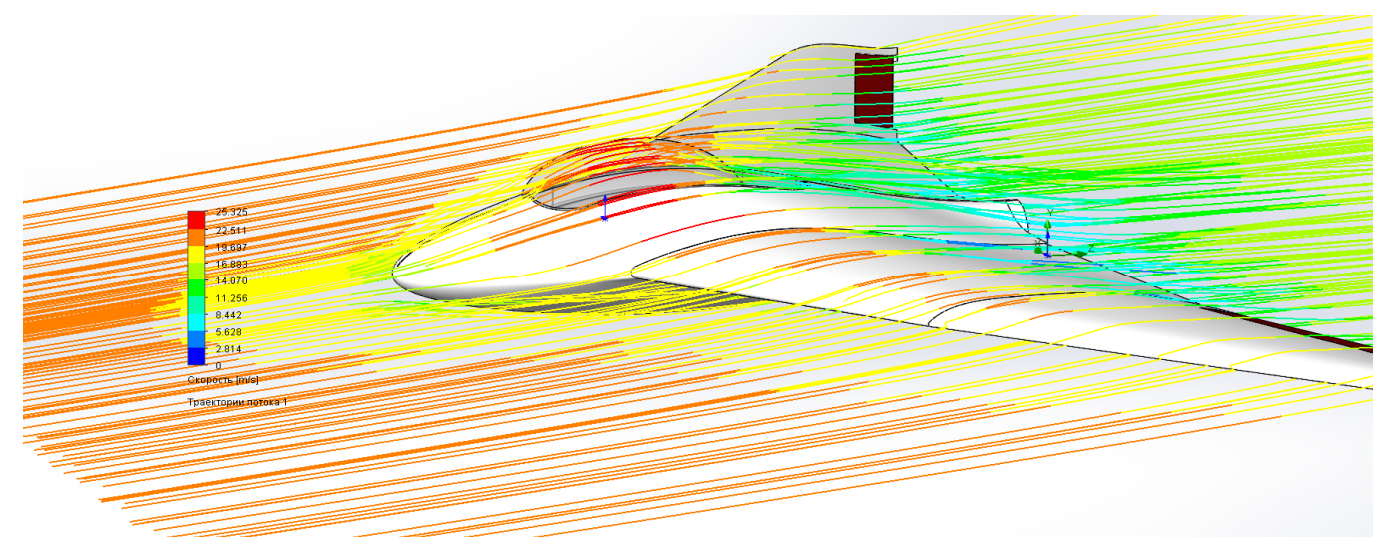

Figure 21: Velocity Distribution of Air Current Lines of Project 6

For global goals, data tables were created for the values of interest for each of the projects and as a result, a common comparative table 1 was formed, which is given below.

Table 1: Comparative Characteristics of Parameter Changes

\begin{tabular}{|c|c|c|c|c|c|c|}
\hline & Project 1 & Project 2 & Project 3 & Project 4 & Project 5 & Project 6 \\
\hline The Lifting Force Fl, N & 83,37 & 100,22 & 94,16 & 89,28 & 94,23 & 85,97 \\
\hline Resistance force Fr, N & 6,26 & 0,17 & 6,38 & 7,25 & 4,29 & 6,25 \\
\hline Pitch Moment Mx, N*m & 10,25 & 11,01 & 11,02 & 10,14 & 11,10 & 10,29 \\
\hline The air temperature, K & 263,06 & 263,09 & 263,07 & 263,08 & 263,08 & 263,09 \\
\hline
\end{tabular}

From the data of the table, it can be concluded that the parameters of air temperature change when changing the aerodynamic shape cannot be taken into account, because the change occurs only by hundredths of a degree.

Analyzing the table dependence, it can be concluded that the latest modification, presented as project 6 , practically does not make changes in the moments of pitch forces and drag forces Fr and lifting Fl forces. In some cases, the drag force, as expected, increased, which can be observed in the visualization of air flows around the airframe. Interestingly, behaves lift $\mathrm{Fl}$, which slightly increased by $20 \%$ after the first modification, but then gradually decreased to $86 \mathrm{~N}$. But the most significant results of the study are changes in the moments of forces - Mx, My, Mz - acting on the projected airframe. The pitch moment Mx about a horizontal axis perpendicular to the direction of movement of the airframe was changed (and by how much?) by increasing the drag Fr, but it can be compensated by deflecting the flaperons within up to 5 degrees before their significant contribution to the drag force occurs, which will entail some change in aerodynamic shape. Changing the design geometry and shape will ultimately affect the duration of the flight. These 
changes will reduce the flight time within $15-20 \%$.

Thus, we have proven that at an angle [alpha] $=6$ degrees, the characteristic of the horizontal flight of most aircrafts, the latest modification with a single-lamp aerodynamic profile is the most optimal for the implementation of our project.

Let us explore the latest modification and see how the lift Fl and drag force Fr behave depending on the angle of attack. We will vary it from 0 to 10 degrees.

The constructed graphs of dependencies are presented in figures 22-24.

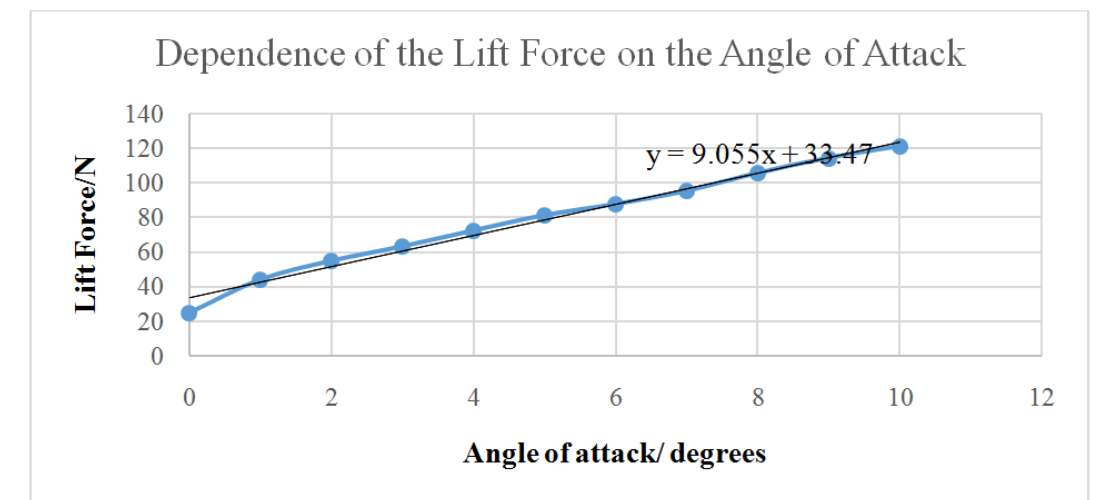

Figure 22: Graph of the Dependence of the Lift Force (in N) on the Angle of Attack (in ${ }^{0}$ ).

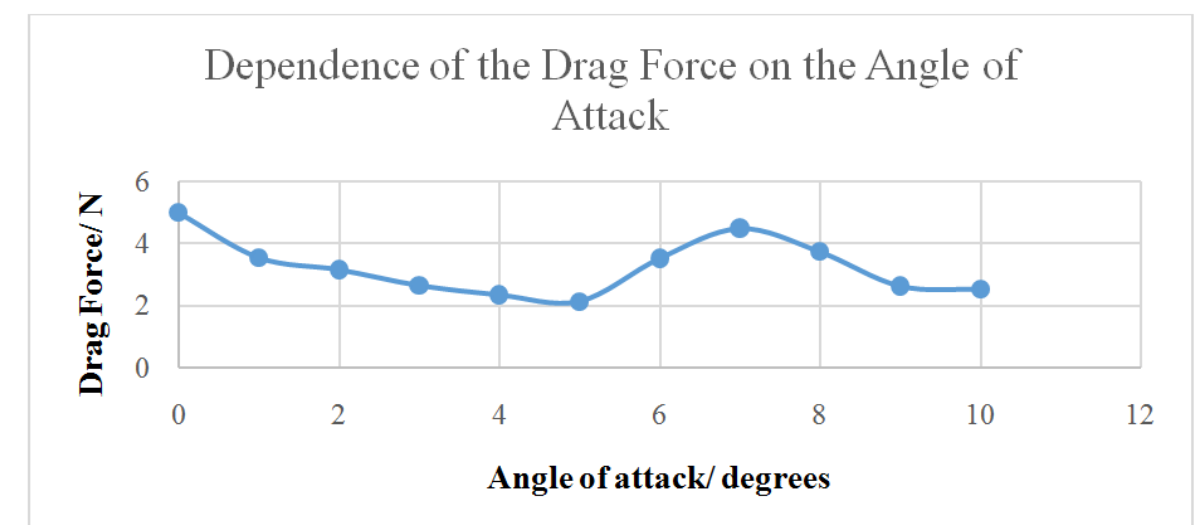

Figure 23: Graph of the Dependence of the Drag Force (in N) on the Angle of Attack (in ${ }^{0}$ ).

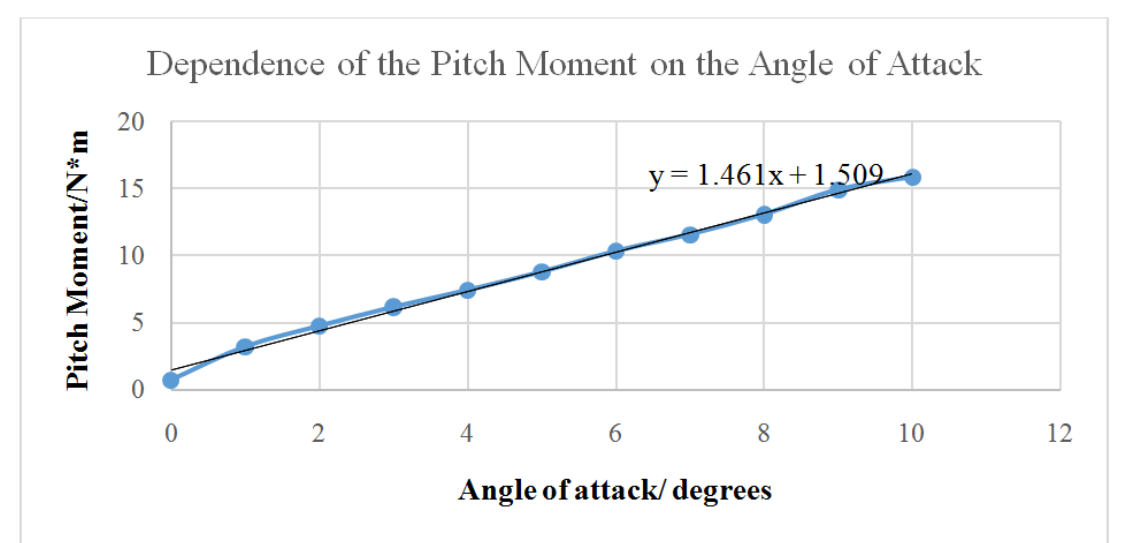

Figure 24: Graph of the Dependence of the Pitch Moment (in N*m) on the Angle of Attack (in ${ }^{0}$ ) 


\section{CONCLUSIONS}

As can be seen from the graphs, with an increase in the angle of attack [alpha] from 0 to 10 degrees, the lift FL and the moment of pitch Mx increase practically, as predicted in the work (Fundamentals of aerodynamics and flight dynamics, 2010). It is also concluded that the aerodynamic profile of the proposed model 6 (figure 9) is not symmetrical, because the graph of the lift dependence (figure 22) does not start from the origin (not from the point $(0,0)$ ). The pitch moment (figure 24) also grows from 3 to $16 \mathrm{~N}^{*} \mathrm{~m}$ with increasing angle of attack from 1 to 10 degrees, almost linearly. This process is explained by the fact that the difference in the total forces applied to the nose and tail parts of the airframe increases due to the different surface area. Special attention should be paid to the result that we see in figure 23 , where there is a clear minimum resistance force, $\mathrm{Fr}=2.14 \mathrm{~N}$, at the angle of attack [alpha] $=5$ degrees, in contrast to the standard values of the angle of 60 degrees. In this regard, it can be concluded that the addition of the upper fairing led to a change in the airframe fairing, which must be taken into account when changing the autopilot programs.

\section{REFERENCES}

1. Anderson, Jr., J. D. (2016). Fundamentals of Aerodynamics Fifth Edition. New York: McGraw-Hill Education.

2. Baldwin B. and Lomax, H. (1978). Thin layer approximation and algebraic model for separated turbulent flows. AIAA Paper 78-257. Reston: AIAA.

3. Belotserkovsky, O. M., Andrushchenko, V. A. and Shevelev, Yu. D. (2000). Dynamics of spatial vortex flows in inhomogeneous atmosphere. Moscow: Janus-K.

4. Belotserkovsky, O. M., Maksimov, F. A. and Shevelev, Yu. D. (2001). 'Spatial problems of computational Aerohydrodynamics". In VIII All-Russian Congress on Theoretical and Applied Mechanics, Perm, Russia, 23-29 August 2001, 93. Perm: Institute of Continuum Media Mechanics of the Ural Branch of the Russian Academy of Sciences.

5. Belov, I. A. and Isaev, S. A. (2001). Modeling of turbulent flows. Textbook. SPb.: Balt. state tech. UNT.

6. Fundamentals of aerodynamics and flight dynamics. (2010). Methodical manual. Riga: Institute of transport and communications.

7. Jagadeesh, V., \& Sandeep, J. Aerodynamic Study of Span Wise Morphing Wing.

8. MacCormack, R. W. (1969). The effect of viscosity in hypervelocity impact cratering. AIAA Paper 69-354. Reston: AIAA.

9. Ministry of Defense of the Republic of Kazakhstan official resource. Retrieved from https://www.mod.gov.kz/rus/dokumenty/voennaya_doktrina/

10. Savinkin, V. V., Solodovnik, A. A. and Kryuchkov, V. N. (2018). Monitoring of the earth's upper atmosphere using unmanned aerial vehicles. Journal of the Eurasian Union of Scientists, 11(56), part 8, 25-30.

11. Shevelev, Yu. D. and Maksimov, F. A. (2000). Numerical simulation of flow near a triangular wing with sharp edges. Novoe V chisl. modeling. Ser. Cybernetics. Moscow: Nauka, 178-209.

12. Shevelev, Yu. D. and Maksimov, F. A. (2003a). Numerical modeling of three-dimensional spatial supersonic flows of viscous gas with flow separation. Matem. modeling. Prob. and results. Ser. Informatics. Moscow: Nauka, 384-421.

13. Jeyan, J. M. L., Nair, K. S., \& Nair, K. S. The Low Speed Aerodynamic Analysis of Segmental Wing Profile.

14. Shevelev, Yu. D. and Maksimov, F. A. (2003b). "The numerical modeling of 3'D laminar and turbulent separated flows”. In Turbulence, Heat and Mass Transfer 4. Proc. Fourth Int. Symp. Turbulence, Heat and Mass Transfer, Antalya (Turkey): 12- 
17 October, 2003, edited by K. Hanjalić, Y. Nagano and M. J. Tummers, 739-746. New York, Wallingford (UK): Begell House, Inc.

15. Tulapurkara, E. G. (1997). Turbulence models for the computation of flow past airplanes. Progr. Aerospace Sci., 33, 71-165.

\section{AUTHORS PROFILE}

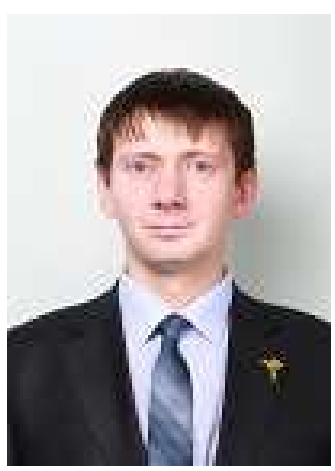

Vasily Nikolaevich Kryuchkov, $\mathrm{PhD}$ student at the Department of Transport and Mechanical Engineering of the North Kazakhstan State University named after M. Kozybaev, Master of Science.

Research interests: mechanical engineering, mathematical modeling of non-stationary processes, research of the upper atmosphere and outer space. He also contributed in publish of more than two research articles.

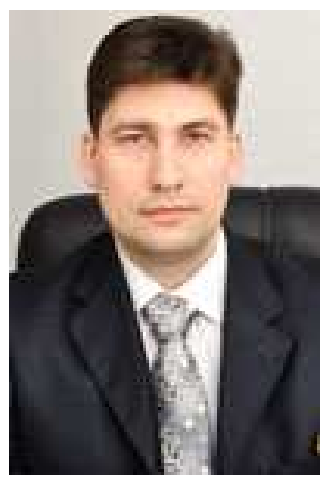

Vitaliy Vladimirovich Savinkin, Assistant professor, Head of the Department of Transport and Mechanical Engineering of the North Kazakhstan State University named after M. Kozybaev. Doctor of Technical Sciences. Authored over 100 scientific publications, of which over 50 articles in journals recommended by the Education and Science Monitoring Committee of the Republic of Kazakhstan, over five invention and utility model patents, five articles in international peerreviewed journals, indexed in Scopus and Web of Science.

Research interests: mechanical engineering, transport, energy, materials science.

Has experience in managing research projects, funded by the Ministry of Education and Science of the Republic of Kazakhstan. H-index = 1. Researcher ID (WoS) AAH-1020-2019. Author ID (Scopus) 0000-0002-7748-3720. Academic supervisor for five $\mathrm{PhD}$ students in the specialty 6D071200 "Engineering" ("Energy-Efficient Technologies in Energy and Engineering"). In 2019, by the order of the Ministry of Education and Science of the Republic of Kazakhstan was appointed a member of the editorial board of the scientific technical journal "Metrologiya". Since 2017, a member of the editorial board of the European Journal of Materials Science and Engineering (EJMSE) published by the Gheorghe Asachi Technical University of Iasi (Romania), indexed in Scopus. He also contributed in publish of more than five research articles. 


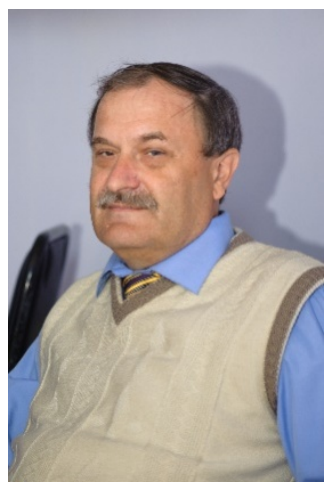

Andrey Andreevich Solodovnik, Professor at the Department of Physics of the North Kazakhstan State University named after M. Kozybaev PhD in Physics and Mathematics, astronomer.

Research interests: physics of the upper atmosphere of the Earth, nature, genesis, and evolution of silvery mesospheric clouds, solar-terrestrial communications, meteor events in the Earth's atmosphere, short-term phenomena on the moon, nature of long-period variable stars, practical astrophysics, philosophical problems of correlation in the system "man Universe".

Corresponding Member of the Kazakhstan Academy of Natural Sciences. Medal "For contribution to the development of the space industry of the Republic of Kazakhstan" (2018). He also contributed in publish of more than five Research articles.

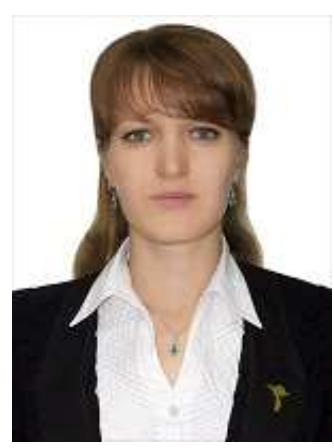

Lyudmila Viktorovna Kryuchkova, PhD student at the Department of Information and Communication Technology of the North Kazakhstan State University named after M. Kozybaev, Master's Degree in Information Systems (2010).

Research interests: mathematical modeling, information security. 